\title{
Changing patterns in suicide among young people
}

\author{
Laurence J. Kirmayer MD
}

See related research article by Skinner and McFaull on page 1029 and at www.cmaj.ca/lookup/doi/10.1503/cmaj.111867

$\mathrm{S}$ uicide is one of the leading causes of death among adolescents and young adults worldwide and is a central public health concern in Canada. Effective prevention requires a clear understanding of the size of the problem, its distribution in the population, and the major risk and protective factors at the levels of the individual, the family and the community.

In the accompanying article, Skinner and McFaull present analyses of mortality data showing that the pattern of suicide among young people in Canada has changed in recent decades. ${ }^{1}$ Over the last 30 years, there has been a modest reduction in suicide mortality among Canadians aged 10-19 years, from 6.2 per 100000 in 1980 to 5.2 per 100000 in $2008 .{ }^{2}$ However, during the same period, there has been an increase in the proportion of female children and adolescents dying by suicide and a change in the methods most frequently used. These data raise questions about the determinants of suicide with implications for prevention.

Skinner and McFaull analyzed mortality data from Statistics Canada for the period 1980 2008, for children (ages 10-14 years) and adolescents (ages 15-19 years). There was an average annual decrease of $1 \%$ in the overall suicide rate; however, there were important differences when the data were stratified by age and sex. There was no change in suicide rates among boys, and male adolescents showed a decrease in suicides at an average annual rate of $2 \%$, from 19.0 to 12.1 per 100000 during the study period. For girls, there was a $50 \%$ increase in the suicide rate during the study period, from 0.6 to 0.9 per 100 000; the rates for female adolescents underwent a larger increase during the same period (from 3.7 to 6.2 per 100000 ). ${ }^{2}$

In addition to these changes in rates of suicide, there were shifts in the profile of the methods used. The use of firearms decreased, and suffocation became the most common method of suicide for children and adolescents.

These results are consistent with data from other countries. A study in the United States covering the period from 1992 to 2006 found a decline of $23 \%$ in suicide rates among young people aged 10-24 years. ${ }^{3}$ That study also found similar changes in the methods used, with a reduction in the use of firearms, no change in poisoning and a shift toward more frequent use of suffocation. A study in Finland found similar reductions in rates for boys and male adolescents and an increase for girls and female adolescents, with an increase in suffocation but no change in the use of firearms. ${ }^{4}$ These cross-national comparisons suggest both local and global processes are at work.

The increase in suicide rates among girls and female adolescents may reflect an increase in the use of more lethal methods for suicide attempts by this group. This change may be specific, or it may reflect some broader convergence in male and female gender roles, although there are clearly great differences in this regard across cultures. ${ }^{4}$

The reduction in the use of firearms for suicides in Canada may reflect the positive impact of legal efforts to restrict access to these weapons as part of injury-prevention strategies. ${ }^{5}$ The increase in suffocation is more difficult to explain. Because hanging, strangulation and other forms of suffocation are grouped together as a single code in the International Statistical Classification of Diseases and Related Health Problems, 10th revision (ICD-10), it is not possible to determine the specific method of suicide from these data. The authors speculate that the increasing popularity of the "choking game," which involves self-induced asphyxiation to achieve a euphoric feeling, may have resulted in accidental deaths misclassified as suicides. However, there is no clear evidence of the frequency

\section{KEY POINTS}

- Suicide rates among Canadian male adolescents have fallen in recent years, but they have risen for girls and female adolescents - trends that are reflected in other Western countries.

- The increase in suicides among girls and female adolescents may reflect an increase in their use of more lethal methods for suicide attempts, such as hanging, strangulation and other forms of suffocation.

- The social and economic deprivation of a region is associated with higher rates of suicide - adversities that are particularly relevant for Aboriginal youth, who are over-represented in Canadian suicide statistics. 
of this misclassification and, in the US at least, there has been no increase in the rate of accidental death by asphyxiation as might be expected. ${ }^{3}$

Adolescents who report experimenting with the "choking game" do have higher rates of attempting suicide. ${ }^{6}$ Recently, we have seen an increase among adolescents of other forms of nonsuicidal self-injury (e.g., cutting), which may also be associated with increased risk of suicide for this group. ${ }^{7}$ The Internet has served as a vehicle for spreading ideas about nonsuicidal forms of self-harm, as well as explicit information on methods of suicide. Although most young people who engage in nonsuicidal self-injury or risktaking behaviours are not suicidal, social networking websites and discussion forums may expose them to information about suicide, increase suicidal ideation in vulnerable people and influence the choice of method among those who ultimately make suicide attempts.

Of course, suicide is not evenly distributed in the population, and these general statistics may mask substantial regional and demographic differences that suggest important social determinants. For example, social and economic deprivation in a region is associated with higher rates of suicide. ${ }^{8}$ Economic inequities may expose young people to a wide range of stressors and negative life events in their families and communities, as well as diminish their own hopes and expectations for a positive future with meaningful opportunities for work and life. The Internet also may play a role by exposing young people to vivid images of autonomy, mobility and conspicuous consumption that intensify feelings of relative deprivation.

These adversities are particularly relevant for Aboriginal youth in many communities across Canada. Both male and female Aboriginal youth die from suicide at rates much higher than their non-Aboriginal counterparts. ${ }^{9}$ Hence, Aboriginal youth are likely over-represented in these numbers. Indeed, given that more than $5 \%$ of the general population aged 10-19 years are Aboriginal, and that the rate of suicide among Aboriginal youth averages from three to five times that of their non-Aboriginal counterparts, ${ }^{9}$ up to $25 \%$ of the total deaths by suicide in this age group may be attributed to Aboriginal youth. Moreover, because the Aboriginal population has a higher birth rate, the proportion of young people in the total population who are Aboriginal has been increasing in recent decades. Although some Aboriginal communities have followed the national trend with declining suicide rates, others have continued to suffer from high rates and have even shown increases during this same period. Thus, any trends seen in the national data for young people will be increasingly influenced by changes specific to the Aboriginal segment of the population.

Although the overall rates of suicide are high, there are wide variations across Aboriginal communities, with many showing rates lower than the general population. Disaggregating the national data to examine regional differences in suicide rates may shed light on processes of resilience unique to Aboriginal communities. ${ }^{10}$ Although such an analysis will be limited by small numbers, it may identify ways to address the persistent adversities that challenge Aboriginal communities and other groups facing structural inequality and economic marginalization. The results will have implications not only for Aboriginal people, but for our approach to suicide prevention more generally. Understanding the impact of these larger social determinants on young peoples' identities, resilience and well-being may hold the key to future reductions in suicide.

\section{References}

1. Skinner R, McFaull, S. Suicide among children and adolescents in Canada: trends and sex differences, 1980-2008. CMAJ 2012; 184:1029-34.

2. Public Health Agency of Canada. Analysis of Statistics Canada mortality data. Ottawa (ON): The Agency; 2012.

3. Bridge JA, Greenhouse JB, Sheftall AH, et al. Changes in suicide rates by hanging and/or suffocation and firearms among young persons aged 10-24 years in the United States: 19922006. J Adolesc Health 2010;46:503-5.

4. Lahti A, Rasanen P, Riala K, et al. Youth suicide trends in Finland, 1969-2008. J Child Psychol Psychiatry 2011;52:984-91.

5. Gagne M, Robitaille Y, Hamel D, St-Laurent D. Firearms regulation and declining rates of male suicide in Quebec. Inj prev 2010;16:247-53.

6. Brausch AM, Decker KM, Hadley AG. Risk of suicidal ideation in adolescents with both self-asphyxial risk-taking behavior and non-suicidal self-injury. Suicide Life Threat Behav 2011;41: 424-34.

7. Wilkinson P, Kelvin R, Roberts C, et al. Clinical and psychosocial predictors of suicide attempts and nonsuicidal self-injury in the Adolescent Depression Antidepressants and Psychotherapy Trial (ADAPT). Am J Psychiatry 2011;168:495-501.

8. Burrows S, Auger N, Roy M, et al. Socio-economic inequalities in suicide attempts and suicide mortality in Quebec, Canada, 1990-2005. Public Health 2010;124:78-85.

9. Kirmayer LJ, Brass GM, Holton TL, et al. Suicide among Aboriginal people in Canada. Ottawa $(\mathrm{ON})$ : Aboriginal Healing Foundation; 2007.

10. Chandler MJ, Lalonde CE. Cultural continuity as a moderator of suicide risk among Canada's First Nations. In: Kirmayer LJ, Valaskakis G, editors. Healing traditions: the mental health of Aboriginal Peoples in Canada. Vancouver (BC): University of British Columbia Press; 2008. p. 221-48.

Affiliations: Laurence J. Kirmayer is with the Department of Psychiatry, McGill University, and the Jewish General Hospital - Lady Davis Institute for Medical Research, Montréal, Que. 